\title{
The Acceptance Intention of Consumers for a Dynamic Payment Mechanism for Health Insurance Coverage
}

\author{
Chie-Bein Chen, Wen-Tsung Wu, Chen-Hua You \\ National Dong Hwa University, Hualien, Taiwan
}

\begin{abstract}
Currently, a consumer's monthly premium payment amount remitted to the National Health Insurance is based on the "monthly real wages," while commercial health insurance uses "consumer age" as the basis for the premium amount charged. In reality, health, salary, and age have no visible connection. Therefore, the insurance premium scheme using salary and age as standards should be improved and adjusted upon. This study uses the Decomposed Theory of Planned Behavior as the research basis, and through the designed questionnaire, investigates the health data gathered from wearable devices and uses big data to process the constructed health assessment indicators. These indicators will be used to analyze whether consumers are willing to contribute to their health insurance using the dynamic payment mechanism. Subsequently, empirical research was performed using hypothesis architecture and structural equation.
\end{abstract}

Keywords: wearable device, health management, health insurance, big data, Decomposed Theory of Planned Behavior

\section{Introduction}

As technology continues to progress, wearable devices have become an important area of study in the industries of electronics, manufacturing, healthcare service, and other related fields. In recent years, due to increased awareness in personal health care as well as in sports and fitness, consumers are attributing more value toward their personal health, and the use of wearable health-tracking devices is increasingly being focused upon in medical care. Many in the technology industry see business opportunities in the aging sector of society, and they focus their innovation on wearable devices, developing different smart phones, smart wristbands, smart clothing and shoes with functionalities such as step counters, activity monitors, and heart rate monitors. Wearable devices can gather data for long periods of time, and then send these data to the cloud, providing the analyzed data back to the users in a short time, so that the users can easily and accurately know the status of their health. Therefore, the built-in physiological signal-sensing device has become the main objective of the wearable device manufacturers. It follows then, that the use and promotion of wearable devices and big data will enable the health management units to better design public health assessment indicators.

Chie-Bein Chen, Ph.D., Department of Business, National Dong Hwa University, Hualien, Taiwan.

Wen-Tsung Wu, Ph.D. student, Department of International Business, National Dong Hwa University, Hualien, Taiwan.

Chen-Hua You, MBA, Department of Business Administration, National Dong Hwa University, Hualien, Taiwan.

Correspondence concerning this article should be addressed to Chie-Bein Chen, 1, Sec. 2, Da-hsueh Rd., Shou-fen, Haulien, Taiwan. 
The monthly premium payment amount a consumer remits to the National Health Insurance is based on the "monthly real wages," while the commercial health insurance uses "consumer age" as the basis for the premium amount. In reality, health, salary, and age have no visible connection. For example: a 50 year-old adult's health is not necessarily worse than a 40 year-old adult's health. On the other hand, merely relying on a person's salary as a basis for determining his health insurance fee is not reasonable. Therefore, this study hopes to understand the health data gathered from wearable devices, and to use the health assessment indicators constructed from the big data to analyze the consumers' acceptance intention in using the dynamic payment mechanism for health insurance costs, thereby explaining any areas that are not aligned with the non-users' concept in relation to the country's current insurance payment. This leads to rising health insurance costs for the people, especially as commercial health insurance requires its consumers to be sick or even deceased in order to process their claims, as shown in Figure 1.

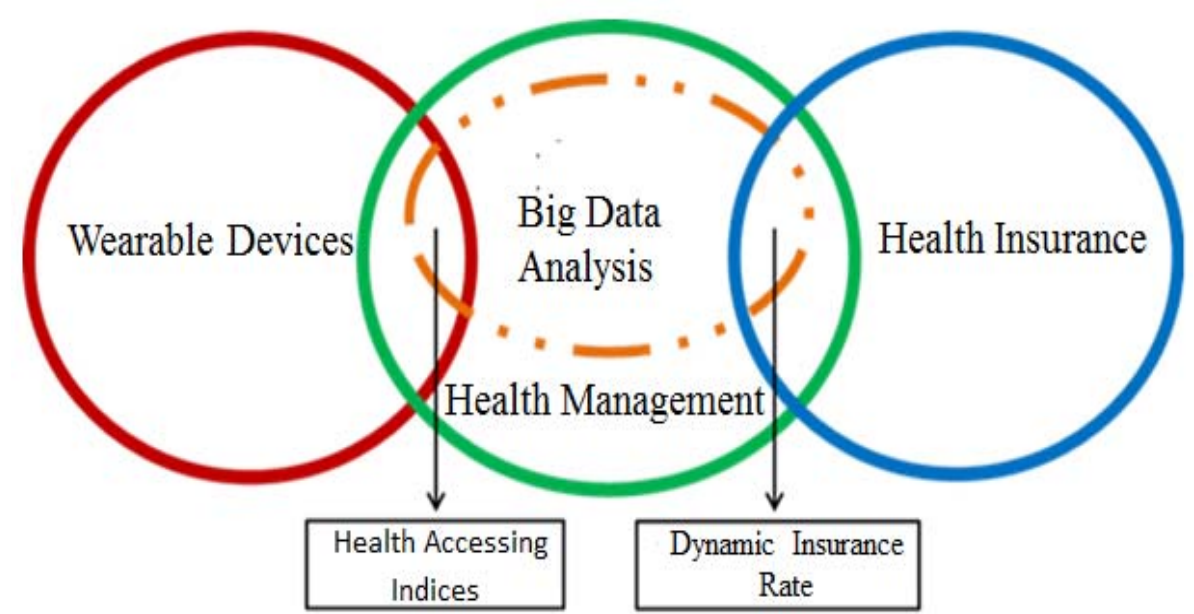

Figure 1. Payment mechanism relationship diagram for wearable devices, health management and dynamic health insurance.

\section{Literature Review}

A wearable device system refers to a product that can be worn by the consumers, and the more convenient it is, the better its utility. Consumers can use the product's functionalities to interact with it, and this type of product can be a communication product (real time, recorded or reproduced), recorder (voice, video or others), or media (operated or remote-controlled), and allow the consumer to closely monitor personal movements, interpersonal relationships, health treatments and others (Bai, You, \& Chu, 2013). In general: (1) " $3 \mathrm{C}$ products can be directly designed to be worn and used by the body" (for example: intelligent eyeglasses, intelligent watches, etc.); or (2) "Integrate the electronic device or functional design into the clothing, so that these become specialized clothing with electronic functionalities" (for example: collar or hat with speaker, a helmet with headset, clothes with embedded device wire and controls, clothes with LED light functionality, etc.), and devices with these features these can be logically considered as wearable products or design (Huang, 2013).

In the 2012 research conducted by the Intelligent Maintenance Systems Research, it was pointed out that in 2011, there were approximately 14 million wearable smart devices sold throughout the world. And in 2016, it is estimated that the total number of wearable devices sold will reach 925 million, and total sales will exceed 
six billion yuan. It is estimated that from 2011 to 2016, the compound annual growth rate can reach $50 \%$ or higher. Given this market trend, the wearable medical device is expected to be the new pattern of health management in the future, and can be used by patients for many different circumstances. Today, in the areas of health care and treatment, wearable smart devices are primarily used for monitoring continuous blood sugar and heart rate; similar devices include intelligent automatic drug delivery device, such as the automatic drug delivery device combined with the continuous blood sugar monitor, or the automatic transmission device for pain relief drugs (Hsieh, 2014).

In 2014, during their developers' conference, Google unveiled their new health application platform, Google Fit, which manages and integrates the user's health data incoming from different wearable devices. This platform architecture uses Google's Fitness Store as their central cloud database, and stores the health/physiological data transmitted among the devices. The developer can transmit the collected data to the Google cloud database, and the users can retrieve from one location their related data gleaned from different devices (Gilbert, 2014).

In 2014, during their developers' conference, Apple also unveiled their health platform, HealthKit, which manages health-related data. Data from different wearable devices can be transmitted to this platform's shared data, and users can also search for and manage their data through this uniform interface. The Apple Health app stores all the data in a unified storage location, and the users can search for their personal physiological data, including heart rate, blood sugar, calorie consumption, sleep, nutrition and other such data, and it can even create emergency information cards such as blood type and allergies to medications. Currently, Apple is collaborating with Duke University to help cancer patients use HealthKit to monitor their blood pressure and weight data. At the same time, through user authorization, the different health or fitness apps can share data, so that physicians, nutritionists, or fitness coaches can also obtain these physiological data, which will enable them to provide more integrated analysis and medical applications (Buhulu, 2014).

In 2014, Compal Electronics and Chang Gung University launched four types of smart clothing, and their functionalities include various types of sensors that can measure the wearer's ECG signal, breathing, temperature, sweat, walking posture, hunching, and falling. It conducts 24-hour monitoring care and continuous recording, and through phone and computer tracking, when there is a change in one of the monitored signals that can lead to a major disease or death, it will immediately send a warning to the wearer ( $\mathrm{Li}, 2014)$.

Health management is a combination of the words "health" and "management". When the World Health Organization (WHO) was established in 1946, its constitution defined health as: "... a state of complete physical, mental, and social wellbeing and not merely the absence of disease or infirmity." By this definition, we can safely say that no such perfectly healthy person exists in this world. Perhaps with the consideration that the 1946 definition is not feasible, the WHO revised the definition of health in the "Ottawa Charter for Health Promotion" released in 1986 during the First International Conference on Health Promotion as: “.... resource for everyday life, not the objective of living. Health is a positive concept emphasizing social and personal resources, as well as physical capacities" (World Health Organization, 1946).

Specifically, health management focuses on health requirements and is the process of planning, organizing, directing, coordinating, and controlling the health resources. In order to plan, organize, direct, coordinate, and control individual and group health, there is a need for comprehensive monitoring, analysis, and evaluation to grasp the individual and group health status. And in order to maintain and protect individual and group health, 
then there is a need to determine the health risk factors, so as to be able to provide health advisory and guidance and to mitigate the health risk factors. The health requirements here can refer to a type of health risk factors, such as high blood, obesity; they can also refer to state of health, noting the presence of diabetes or Alzheimer's disease. Health management methods can include performing analysis on the health risk factors, performing quantitative evaluation on the health risks, or providing supervision and guidance during the intervention process (Huang \& Chen, 2007).

The earliest appearance of the health management concept occurred in the United States during the 1950s. Currently, for the insurance industry, through the disease prevention management being done by the insured individuals, the probability of insured individuals getting sick and the amount of medical bills had gone down, thus reducing health insurance payments by the insurance organizations. It can be seen that health management and the insurance industry are naturally related. Following the degradation of the natural environment and the complication of the social environment, however, disease factors have begun to increase in number and complexity, and the general population does not have the ability to identify the health risk factors. Therefore, there is a need to engage professionals and organizations to provide specialized health guidance (Pan, 2014).

Based on research data from the US-based Wellpoint Company, $7 \%$ of clients will consume $70 \%$ of health care costs, and most of these costs were incurred in paying for the treatments of cardiovascular and cerebrovascular diseases, diabetes, and other chronic diseases; if managed through health insurance companies, however, the hospitalization rate and average length of stay of chronic disease patients clearly went down.

Second, for every one yuan invested in health management, it can produce eight yuan return on investment, thus substantially reducing the health care costs that health insurance companies incur, and also substantially improve the companies' financial return. Health management can increase the insurance industry's core competitiveness, and as for the health insurance companies, through preserving the clients' good state of health, the health care costs and health insurance premiums will go down. At the same time, as insurance companies provide targeted health management services to their clients, it will not only increase the effectiveness of health management, but will win the clients' trust and loyalty (Li, 2011).

In 2014, Cathay Life Insurance first proposed that "Insurance can be linked to health management services," and aside from insurance policies such as "high-volume" of hospitalization, inpatient surgery, and outpatient surgery health insurance protections, during the insured period, the insured party can even receive specially designed services from Cathay Healthcare Management. There are professional health care teams who focus on the clients' health status to design a health plan, and also provide scheduled specialist consultations, nutritional counseling, and exercise guidance. The clients can even use their mobile phones to upload pictures of meals, with professional nutritionist tracking their diet. Aside from this, clients can also use claims to directly offset medical expenses when staying at Cathay Hospital and other accredited facilities, thus reducing the burden of financing health care costs (Chuang, 2014). In 2014, Nan Shan Life Insurance and Taipei Medical University worked together to invest annual premiums of five million yuan to be used in remote health care service. This service combines cloud technology and convenient measuring instruments, so that users can continuously manage their health, and can effectively control and minimize the onset of chronic diseases. This move not only increases clients' knowledge of health management, but can also help incorporate together the insurance industry and the field of preventive medicine. Aside from this, remote health care services can also 
help clients arrange for emergency and notify emergency contact person, so that the clients can receive effective care during emergency situations. In the future, there also be the latest wearable devices, that will provide comprehensive, constant recording of physiological data, helping clients to manage their health more easily (Chang, 2014).

In the age of internet computing technology, the interaction of wearable devices and the surrounding infrastructure can produce large amount of data, and by analyzing and organizing the constructed indicators, this interaction will enable the general public to have a grasp of their own health condition anytime.

(1) The required parameters to be measured for the most common chronic diseases (Villasenor, 2014) are:

(a) Blood pressure

For most primary and secondary individuals with hypertension, the usual required measurements are systolic blood pressure, diastolic blood pressure, and heart rate, which are measures used to evaluate blood pressure stability. The current sensors in these devices use cuff pieces, but since they cannot continuously monitor the numbers they leave a large margin of error. Fortunately, cuff-less sensor technology is currently being developed. For example: wearable photoplethysmography signaling device with height sensors can greatly help affected individuals measure their blood pressure without time or spatial constraints.

(b) Blood sugar

To those suffering from insulin and non-insulin dependent diabetes mellitus, if measurements of blood sugar are taken multiple times daily, then they can achieve continuous monitoring, helping them control their illness and drug treatments. Industry experts have already developed the technology for continuously measuring blood sugar, so that diabetics can better control their blood sugar.

(c) ECG

Those with cardiovascular diseases, to measure these parameters usually need two main electrodes to identify the pins and to study the single pin electrode of the ECG to measure disease parameters. For the person who merely wants to measure heart rate, this type of operation is very inconvenient. However, wearable ECG devices are now available in the market; they can integrate the electrodes in the devices, and can capture signal using wireless antenna, which can greatly increase the convenience of measuring these parameters. Other devices such as ring type heart rate sensors are also being developed.

(2) Health assessment indicators in the medical field: body fat percentage, visceral fat, body mass index.

(a) Body fat percentage

Body fat percentage refers to the ratio of fats in body composition. Excessively high body fat percentage is the main trigger for many chronic diseases, and to maintain the body percentage within the normal level, it is not possible to use visual inspection or the usual weighing scales to ascertain the values correctly. Once it is determined that the body fat percentage is too high, then the person will need to do a self-check of blood pressure, blood sugar, liver functions etc., to know if he has any further hidden health risk factors (Zhan, 2008).

(b) Visceral fat

Body fat is divided into subcutaneous fat and visceral fat. Visceral fat is attached to the abdomen, to the adipose tissue on top of the gastrointestinal tract mesentery (to secure the position of the intestinal viscera), which can support and secure the internal organs. There is a significant relationship between increased visceral fat and the rate of occurrence of high-blood pressure, diabetes, hyperlipidemia, and cardiovascular diseases (Kudo, 2010). 
(c) Body Mass Index

The WHO suggested using the Body Mass Index (BMI) to measure the level of obesity, and its formula is weight (kilogram) divided by height (meter) squared. Research has shown that being overweight or obese (BMI $\geqq 24$ ) is the main risk factor of chronic diseases such as diabetes, cardiovascular diseases, and malignant tumors while the health problems associated with being underweight are malnutrition, osteoporosis, and sudden death (Health Promotion Administration, Ministry of Health \& Welfare, 2015).

Currently, the national health insurance of the country can be divided into government-insured national health insurance, and the commercial health insurance sold by life insurance companies. The national health insurance is a health insurance system that has the whole country's citizens as insured parties. When the insured party and his family suffer illness, injury, or fertility problems, then they can avail the appropriate medical services, thus making it a form of self-help and mutual help, as well as a risk sharing social security system.

In the process of organizing the national health insurance, the goal is to provide the nation's citizenry with universal medical care. Therefore, when compared to a society's comprehensive medical insurance needs, universal medical care becomes insufficient. For example, when considering those in prison, whether inmates or security personnel, juvenile department executor, ward fee difference, special nurses, vaccination, medicines and cosmetic surgery, or health risks incurred due to war, earthquakes and other natural disasters, it is not appropriate to invoke national health insurance. This also paved the way for life insurance companies to provide commercial health insurance, in order to meet the society's medical security needs. With the commercial life insurance sold by life insurance companies, when the insured becomes ill or is involved in an accident, all medical and hospitalization fees are shouldered by the insurance companies or via compensation insurance, which can make up for the insufficiency of the national health insurance (Chen, 2000). Currently, the national health insurance uses income as basis for calculating the insurance costs, and the salary benchmark is based on the insured amount table released by the National Insurance Health Administration, Ministry of Health and Welfare, as opposed to the actual salaries earned. The insured amount table is currently divided into 52 levels, where the lower limit is similar to the base salary published by the Council of Labor Affairs.

The "health insurance" mentioned in this study refers to the health insurance being sold by life insurance companies and commercial insurance companies. When the insured becomes ill or is injured in an accident, all the medical and hospitalization fees, as well as the loss of income due to disability, will be shouldered by the insurance companies or by compensation insurance.

According to Li (2004) health insurance is also called medical insurance, and covers specific diseases, surgery, hospitalization, maternity-related medical costs reimbursements. The paid costs can be simply divided into: hospitalization expense insurance, hospitalization medical expense insurance, major medical expense insurance, cancer disease medical expense insurance, surgical medical expense insurance, and daily amount of health insurance paid.

Based on the "Life Insurance Underwriting Practices and Professional Ethics Processing Standards" established by the Life Insurance Management Institute of the Republic of China on July 27, 1995, insurance companies can consider age, sex, health status (medical report, medical history, etc.), family history (family life expectancy, genes...), occupation, financial status, hobbies and leisure activities, life habits (home environment, 
eating habits...), and other life insurance association information as underwriting factors, and these are also factors that determine the insurance premiums that people should pay.

Based on the insured party's risk factors resulting determined by a risk assessment, if the risk goes up above a level's range, then there is a need to add a certain percentage of risk premium, and if the risk exceeds the allowable range, then the process is to either "defer underwriting" or "refuse to insure."

As for those who recently joined the work force, the budget for insurance is usually low. Therefore, in order to solve problem of the policy holder, some sales agents will design "natural rates" policies. The advantage of this type of policy is that for younger consumers, the premium to be paid is much lower than the traditional "level premium" policies. However, there is a disadvantage in that as consumers grow older their premiums increase quickly.

Although the income of working professionals can increase as they grow older and their job grows, but if we assume an algorithm where the rate increases "once every five years" with a one year term medical insurance rider as an example, then the premium increases approximately $20 \% \sim 40 \%$ every five years (there is some difference due to different ages, but general growth is $20 \% \sim 30 \%$ ). For the policyholders holders who are unprepared for this increase, future premium payments can become a significant burden. Aside from this, in underwriting natural rates term health insurance policies, the insurance companies are given the right to adjust the premiums anytime. Even if the highest adjustment is $20 \%$, as time passes, the premiums will become an unbearable burden to the policyholders ( $\mathrm{Li}, 2008)$.

Big data refers to different types of data created constantly and under different types of circumstances, and by retrieving the effective information and performing different cross-analysis, they can be used as important references for marketing and business. At the start, big data is used to understand the clients' appearances, so as to increase the processing efficiency of the organization's internal administration. Currently, big data is used to forecast the clients' needs, and is subsequently used for important references and bases for decision-making (Hu, 2013).

Generally speaking, the big data is defined to include "Volume, Velocity and Variety," but there are people who added two more Vs: Veracity and Value. Regardless of the number of Vs, the unique features of big data information compared to traditional data are: diverse data sources, numerous and complex classifications, non-structured data, and as the data change very rapidly, it results in massive volume of data ( $\mathrm{Li}, 2015)$.

(1) Big Data and Wearable Devices

According to $\mathrm{Su}$ (2014), on June 25, 2014, during their developers' conference, the US search engine giant Google released their new health data platform Google Fit, which can collect personal health data using different high-tech wearable devices. Google Fit allows wearable devices to measure blood pressure, heart rate, and other personal activity data, and upload these data into Google's cloud server.

Drchrono, an electronic medical records company located in Mountain View, California, also developed a health application that can be used in conjunction with Google Glass. The doctor who has the registered Drchrono application, after obtaining the patient's approval, can let the patient wear the glass loaded with the said application, to record the medical diagnosis and procedures. These recorded videos, pictures, and notes can be saved in the patient's electronic medical records.

On September 17, 2014, Apple announced the health suite HealthKit, which can be considered a personal health data platform. It can integrate other third-party health application data, and at the same time, it can 
cooperate with medical institutions, allowing the medical institutions to receive and transmit the patient's data. This suite's partners include Nike, Fitbit, Jawbone, and Runkeeper. Users can view in HealthKit their daily calorie consumption, hours of sleep, number of kilometers run, and other such data. And user's data blood pressure and weight data can be collected and analyzed using iPhone or iPad applications.

On May 29, 2014, Samsung released the wearable health device reference platform Simband health band, which is also referred to as "survey equipment." This wristband is equipped with Wi-Fi and Bluetooth, and can monitor heartbeat, blood sugar, blood pressure, and other data, and it uses Samsung's Architecture for Multimodal Interactions (SAMI) cloud computing platform system to analyze the collected wearable device sensor data, and then shares the related data to the other devices or services (Candice, 2014).

(2) Big Data and Health Insurance

Fang Yuan Jin, Assistant IT Manager of China's Taikang Life Insurance Co. Ltd., pointed out that China's current insurance industry is growing, with personal health insurance showing the most visible growth. Taikang's financial data alone numbered 1.4 billion in 2011, which is a very large amount of data; at the same time, Taikang employs the telephone sales model, where 10,000 employees make a record of 100 calls per day; in addition, they process hundreds of thousands of claims, and the affected policies, medical history and treatment data volume is massive. Clearly, just for one health insurance company, the data it produces reach up to billions.

Because this large amount of unstructured data being produced everyday such as videos, sounds, pictures, texts, product information, and time records likely provide information on the consumers' consumption habits, market changes, product trends, and large amount of historical records, these data are very important to a company and organization's future business and development (Chen, 2012).

From the large amount of consumers' qualitative and quantitative data gained, the insurer can effectively and comprehensively capture the policyholders' profile using data analysis. This step can help the insurer more accurately get a picture of the policyholders' states of being, and can truly convert the policyholders' increasing insurance needs into income. The insurer uses data analysis to increase their knowledge of the policyholders, thus tailoring their marketing strategies, which is a great advantage. This technique first creates the policyholder retention model, performs market segmentation on their clients, so as to effectively retain high-value clients.

By analyzing the policyholders' behavior, insurance content, and the policyholders' subsequent lapse behavior, the policyholder retention model is created, and it gives a score for each policyholder to represent their probability of lapse, where the lower the score, the higher the probability of lapse. And in order to increase the accuracy of the policyholder lapse ratio, the result of the policyholders' social network analysis will be used as the input variable of the policyholder retention model (Chen et al., 2004).

\section{Decomposed Theory of Planned Behavior}

The Decomposed Theory of Planned Behavior (DTPB) was conceptualized in 1995, when Taylor and Todd (1995) used the Theory of Planned Behavior by Ajzen's (1985) and the Technology Acceptance Model by Davis (1989) as foundation, and added Roger's (1983) Diffusion of Innovation Theory, Self-efficacy, reference groups and environmental help concepts.

In Ajzen's (1985) Theory of Planned Behavior, attitude, subjective norm, and perceived behavioral control all use a one-dimensional structure, and the pattern of these one-dimensional structure beliefs can possibly lead 
to the positive and negative beliefs offsetting each other, thus it is unable to consistently identify the subjective norm, behavior and attitude, and perceived behavioral control. Therefore, many scholars have criticized this theory.

Bagozzi (1981) discovered that the Multi-dimensional Belief Structures can be used to describe factors that influenced "behavior and attitude", better than Uni-dimensional Construct. And Shimp (1984) in his research also discovered that the Multi-dimensional Belief Structures could better explain "behavior and attitude."

In view of Taylor and Todd (1995) restructured the one-dimensional beliefs in the Theory of Planned Behavior, namely the "behavior and attitude," "subjective norm", and "perceived behavioral control," into multi-dimensional belief variables, and separately propose the construct combinations of elements. The Theory of Planned Behavior started in 1995 and has been evolving for the last 20 years. When Taylor and Todd (1995) compared the Decomposed Theory of Planned Behavior (DTPB), Theory of Planned Behavior (TPB), and Technology Acceptance Model (TAM), they found that the predictive ability of DTPB is higher than the traditional TPB and TAM, and many local and international scholars have determined through actual research that DTPB is suitable for investigating the usage intention for software or technologies, and the results are more geared towards setting-up. The scholars also discovered in their research that the explanatory power of DTPB is superior to those of other models.

Therefore, this study uses DTPB and has the following advantages: (1) it makes consistent the relationship between the preceding factors and each belief dimension, (2) through the decomposition method, it makes the relationship between the preceding factors and each belief dimension clearer and more easily understood, and (3) it clearly points out specific factors that can influence adoption and use.

\section{Research Methods}

\section{Research Framework}

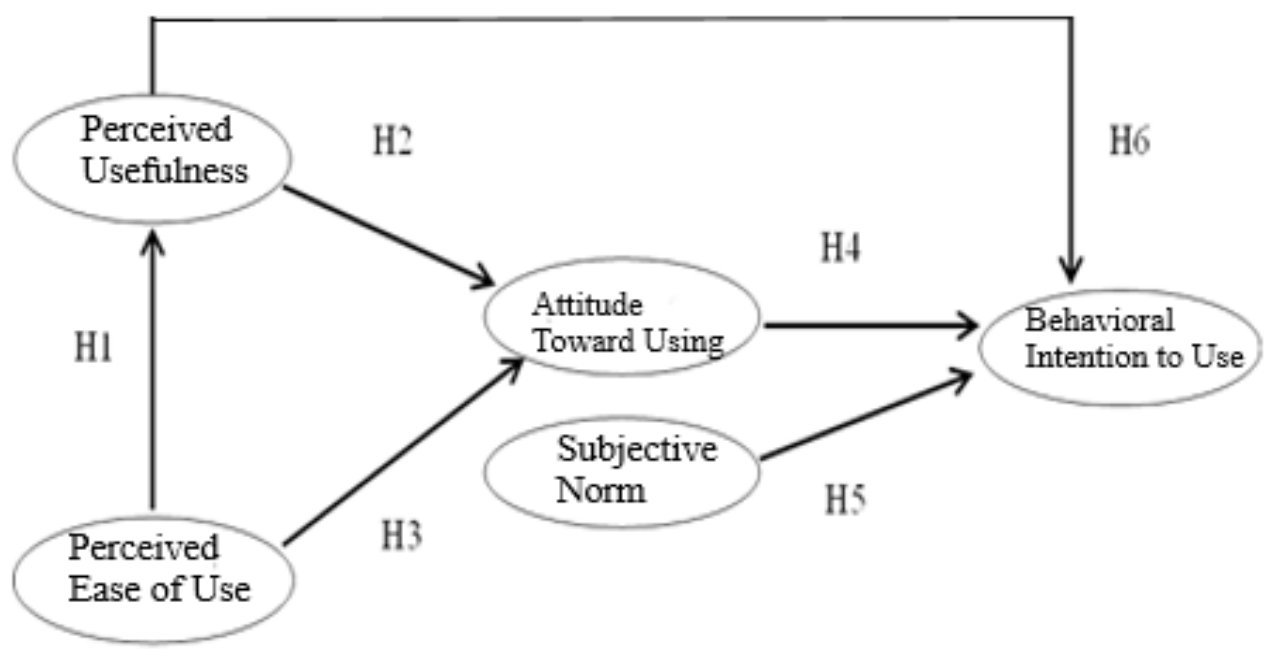

Figure 2. Framework diagram of this study (Davis et al., 1989).

(First oblong top left: Perceived Usefulness; Second oblong lower left: Perceived Ease of Use. Center oblong, top:

Attitude Toward Using. Center oblong, bottom: Subjective Norm. Right oblong: Behavioral Intention to Use)

This study investigates the relationship among consumers' subjective norm, perceived usefulness, perceived ease of use, attitude towards using, and behavioral intention to use. It uses the Decomposed Theory 
of Planned Behavior as research foundation, where the used dimensions include: Subjective Norm (SN), Perceived Usefulness (PU), Perceived Ease of Use (PEOU), Attitude Towards Using (ATU), and Behavioral Intention to Use (BI), to construct this study's framework diagram, as shown in Figure 2.

\section{Sampling Method and Sampling Target}

This study mainly uses the entity questionnaire distribution method to conduct data gathering, the questions and operational definition are created based on research literature of previous related information products, and then modified based on actual situations. Each scale use the seven-point scale score (1 represents "Strongly Disagree", 7 represents "Strongly Agree"), and records five constructs. The research targets are mainly the general consumers. It also aims to infer the influence on each other and relationship among the subjective norm, perceived usefulness, perceived ease of use, attitude towards use, and behavioral intention to use of insurance professionals towards the health insurance dynamic payment mechanism.

\section{Research Hypotheses and Operational Definition Measure}

H1: The user's "perceived ease of use" of the health insurance dynamic payment mechanism has a positive influence on the "perceived usefulness."

Referring to the research done by Davis (1989) and Taylor and Todd (1995), if the user does not have to spend too much time and effort to learn the new technology, but is able to achieve performance improvement, then the user's "perceived ease of use" of information technology will have a positive influence on its "perceived usefulness;" if the user thinks that the health insurance dynamic payment method is easy to use, then the probability of the user thinking that the health insurance dynamic payment method is useful would be higher.

H2: The user's "perceived usefulness" of the health insurance dynamic payment mechanism has a positive influence on the "attitudes towards using."

Referring to the research done by Davis (1989) and Moon and Kim (2001), if the use of information technology can improve one's work performance, then the user would feel that information technology is useful. Therefore, it would also improve the attitude towards using information technology; if the user thinks that the health insurance dynamic payment mechanism is useful, then the user's own "attitude towards use" will also be positively influenced.

H3: The user's "perceived ease of use" of the health insurance dynamic payment mechanism has a positive influence on the "attitude towards using".

Referring to the research done by Davis (1989) and Moon and Kim (2001), if the user spends lesser time and effort in learning how to use information technology, then the user's "perceived ease of use" towards information technology would be positively impacted; if the user thinks that the health insurance dynamic payment mechanism is easy to use, then the user's own "attitude towards use" would be positively influenced.

H4: The user's "attitude towards using" the health insurance dynamic payment mechanism has a positive influence on the "behavioral intention to use."

Referring to the research done by Davis (1989) and Moon and Kim (2001), if the user utilizes information technology to finish his work, and if due to this, the user's behavioral intention to use towards information technology increases, then the user's "attitude towards using" information technology will have a positive influence on his "behavioral intention to use;" if the user has a more positive attitude towards the health insurance dynamic payment mechanism, then the probability of using this mechanism would also be higher. 
H5: The user's "subjective norm" towards the health insurance dynamic payment mechanism will positively influence the "behavioral intention to use."

Referring to the research done by Mathieson (1991), Taylor and Todd (1995), and Chau and Hu (2001), the user's subjective norm towards the research objective behavior has an evident influence on the behavioral intention to use. Because the health insurance dynamic payment mechanism is in the early stages of innovation and growth, this study deduces that the consumer's subjective norm in using the health insurance dynamic payment mechanism will positively influence his behavioral intention to use.

H6: The user's "perceived usefulness" towards the health insurance dynamic payment mechanism has a positive influence on the "behavioral intention to use".

Referring to research done by Davis (1989), Moon and Kim (2001), and Taylor and Todd (1995), the user's "perceived usefulness" of information technology will have a positive influence on his "behavioral intention to use;" if the user feels that the health insurance dynamic payment mechanism is useful, then the probability of using the health insurance dynamic payment mechanism would be higher.

\section{Data Analysis Method}

After the questionnaires from this study were collected, the data were examined, and the effective questionnaires were coded and logged. We used the statistical analysis software package SPSS to perform basic analysis of the questionnaire data, and then used structural equation model software AMOS to perform empirical analysis on the research model's various dimensions of whole model hypothesis. This study performed confirmatory factor analysis to determine each dimension's reliability, convergent validity, and discriminant validity, and then performs fitness assessment of the whole model, thereby validating all of the hypotheses.

\section{Data Analysis}

\section{Descriptive Statistics Analysis}

We selected 73 males and 112 females for our sample. In terms of age, there are 13 who are 25 years old and below, 34 who are 26 to 30 years old, 28 who are 31 to 35 years old, 28 who are 36 to 40 years old, 23 who are 41 to 45 years old, 17 who are 46 to 50 years old, 23 who are 51 to 55 years old, 17 who are 56 to 60 years old, and two who are 61 years old and above. In terms of educational attainment, there is one who attained junior high school level and below, 46 who attained senior high school level/vocational level, 45 who have specialist degrees, and 93 who have college degree. Nineteen of them have 20,000 and below, 65 have 30,000 to $40,000,54$ have 50,000 to $60,000,21$ have 70,000 to $80,000,16$ have 90,000 to 100,000, and 10 have more than 100,000. In terms of residence, 184 live in the northern districts, and one live in the central district. In terms of premiums paid, 20 of them pay 0 to 3,000, 76 have 3,000 to 10,000, 35 have 10,000 to 20,000, 28 have 20,000 to 30,000 , and 26 pay 30,000 and above.

\section{Reliability Analysis}

The Cronbach's $\alpha$ dimension for this study is shown in Table 1, where each factor's Cronbach's $\alpha$ reliability coefficients are: Subjective Norm is 0.694, Perceived Ease of Use is 0.649, Perceived Usefulness is 0.727, Attitude Towards Use is 0.618, and Behavioral Intention to Use is 0.681 .

\section{Validity Analysis}

We can see in Table 2 that, for the model used this study, the measurement error of the model's 
measurement variables does not contain negative values. This study's factor load ranges between $0.512 \sim 0.973$, which shows that the relationship between the latent variables and their corresponding measurement variables has reached a significant level, indicating that the estimation results are in line with the standard. On the other hand, in the area of internal structure fit, in terms of Composition Reliability (CR), the Subjective Norm is 0.705, Perceived Ease of Use is 0.864, Perceived Usefulness is 0.748, Attitude Towards Use is 0.698, and Behavioral Intention to Use is 0.780 , where all of them are above 0.5. In terms of Average Variability of Extraction (AVE), the Subjective Norm is 0.445, Perceived Ease of Use is 0.684, Perceived Usefulness is 0.509, Attitude Towards Use is 0.366, and Behavioral Intention to Use is 0.529 , where most of them are above 0.5. This shows that internal structure fit is in line with the inspection standards. Therefore, it can be seen that this study's architecture theory model has reached the basic standards of fit.

Table 1

Reliability Analysis per Dimension

\begin{tabular}{llll}
\hline Classification & Dimension & Cronbach's $\alpha$ value & \\
\hline & Subjective Norm & 0.694 & In line \\
& Perceived Ease of Use & 0.649 & \\
Insurance practitioners & Perceived Usefulness & 0.727 & \\
& Attitude Towards Use & 0.618 & \\
& Behavioral Intention to Use & 0.681 & \\
\hline
\end{tabular}

Table 2

Sorting Table of Confirmatory Analysis Results of Insurance Practitioners

\begin{tabular}{|c|c|c|c|c|c|c|}
\hline Dimension & Subject & Factor Load & Measurement Error & $S M C$ & $\begin{array}{l}\text { Composition } \\
\text { Reliability }(\mathrm{CR}) \\
\end{array}$ & $\begin{array}{l}\text { Average Variability } \\
\text { Extraction }(A V E)\end{array}$ \\
\hline \multirow{3}{*}{ Subjective Norm } & $X_{12}$ & 0.689 & 0.525 & 0.475 & \multirow{3}{*}{0.705} & \multirow{3}{*}{0.445} \\
\hline & $X_{13}$ & 0.577 & 0.667 & 0.333 & & \\
\hline & $X_{14}$ & 0.727 & 0.471 & 0.529 & & \\
\hline \multirow{3}{*}{ Perceived Ease of Use } & $X_{21}$ & 0.973 & 0.053 & 0.947 & \multirow{3}{*}{0.864} & \multirow{3}{*}{0.684} \\
\hline & $X_{22}$ & 0.765 & 0.415 & 0.585 & & \\
\hline & $X_{24}$ & 0.722 & 0.479 & 0.521 & & \\
\hline \multirow{3}{*}{ Perceived Usefulness } & $X_{31}$ & 0.693 & 0.520 & 0.480 & \multirow{3}{*}{0.748} & \multirow{3}{*}{0.509} \\
\hline & $X_{32}$ & 0.886 & 0.215 & 0.785 & & \\
\hline & $X_{33}$ & 0.512 & 0.738 & 0.262 & & \\
\hline \multirow{4}{*}{ Attitude Towards Use } & $X_{41}$ & 0.549 & 0.699 & 0.301 & \multirow{4}{*}{0.698} & \multirow{4}{*}{0.366} \\
\hline & $X_{42}$ & 0.719 & 0.483 & 0.517 & & \\
\hline & $X_{44}$ & 0.562 & 0.684 & 0.316 & & \\
\hline & $X_{45}$ & 0.577 & 0.667 & 0.333 & & \\
\hline \multirow{3}{*}{$\begin{array}{l}\text { Behavioral Intention } \\
\text { to Use }\end{array}$} & $X_{52}$ & 0.823 & 0.323 & 0.677 & \multirow{3}{*}{0.780} & \multirow{3}{*}{0.529} \\
\hline & $X_{53}$ & 0.639 & 0.592 & 0.408 & & \\
\hline & $X_{54}$ & 0.709 & 0.497 & 0.503 & & \\
\hline
\end{tabular}

Notes. Extraction (AVE) of "subjective norm," "perceived ease of use," "perceived usefulness," "attitude towards use," and "behavioral intention to use" are $0.542,0.680,0.467,0.628$, and 0.450 respectively, and all are above the acceptable level of 0.50 . As a whole, the internal fit of this study's theoretical model is good.

In the statistical test volume of structural fit, the test results are: $\chi^{2} / d . f$. is $1.845, G F I$ is $0.896, A G F I$ is 0.849, RMR is $0.038, R M S E A$ is $0.068, N F I$ is $0.821, C F I$ is $0.907, P N F I$ is 0.643 , and PGFI is 0.619 . The 
statistical volumes are in line with the ideal calibration values. Therefore, it produces good results in overall structural fit, which are shown in Table 2.

\section{Structural Equation Model Analysis}

In the theory's model assessment, Bagozzi and Yi (1988) proposed that it should be assessed using basic fit, internal fit, and overall fit.

Basic Fit - Consists of Three Inspection Standards. 1) The error variance should not be negative; 2) The standardized factor load should not be lower than 0.50 or higher than 0.95 , and it should reach significant levels; 3) The standard errors should not be too large. The measurement model result of the overall theoretical model is shown in Table 2. As shown by the results of this study, the error variances of the theoretical model do not have negative values, the standardized factor load values are not lower than 0.50 or higher than 0.95 , and have reached significant levels, and at the same time, there are no significantly high standard errors. Therefore, as a whole, the basic fit of this study's model has reached an acceptable level.

Internal fit - Consists of three inspection standards. 1) Composition Reliability (CR) should be above 0.6 or 0.5 ; 2) Average Variability Extraction should be above 0.5 ; 3) Whether each item's reliability is above 0.70 or not. The results of the overall theoretical model can be seen in Table 3. The combined reliability coefficient of the overall dimensions for "subjective norm," "perceived ease of use," "perceived usefulness," "attitude towards use," and "behavioral intention to use" are $0.776,0.863,0.778,0.835$, and 0.709 , respectively, and all are above the acceptable level of 0.50 . The Average Variability

Overall Fit-Has to go through the inspection of Absolute Fit Indices, Incremental Fit Indices, and Streamlined Fit Indices.

(1). Absolute Fit Indices: These include $\chi^{2} / d . f$., GFI, GFI, RM, RMSEA

The absolute fit measurement indices of this study's overall theoretical model are: $\chi^{2} / d . f .=1.895, G F I=$ $0.891, A G F I=0.846, R M R=0.037$ and $R M S E A=0.07$. All of the study's indices have met the standards, as shown in Table 3.

(2). Incremental Fit Indices: These include $N F I$ and $C F I$

The incremental fit measurement indices of this study's overall theoretical model are: $N F I=0.812$ and $C F I$ $=0.899$. Both of these have met the inspection standards, as shown in Table 3 .

(3). Streamlined Fit Indices: These include PNFI and PCFI

The streamlined fit measurement indices of this study's overall theoretical model are: $P N F I=0.65$ and $P G F I=0.719$. Both of these have met the inspection standards, as shown in Table 3.

\section{Hypothetical Relationship Verification}

After determining the compatibility of the overall model, we can further determine the estimated values of the assessment measurement model and structural model, and from these, investigate the relationship between measurement variable and latent variable, and also the relationship between latent variables, and then obtain the comparison between the structural relationship of the overall model and the above evaluation standard. This study's theoretical model has reached has reached a certain level of compatibility, thus can continue the model's causality validation to analyze the association among the five variables- "subject norm," "perceived ease of use," "perceived usefulness," "attitude towards use," and "behavioral intention to use." The overall path analysis chart is shown in Figure 3. 
The correlation coefficient of the perceived ease of use on the perceived usefulness is 0.138 , indicating a low correlation, and it shows that the insurance practitioners' perceived ease of use of the insurance dynamic payment mechanism has low correlation with the insurance practitioners' perceived usefulness of the insurance dynamic payment mechanism. Therefore, hypothesis $\mathrm{H}_{1}$ is established. We can discover from Table 4 that, the insurance practitioners' perceived usefulness of the insurance dynamic payment mechanism has a positive influence on the attitude towards use, where its standardize regression coefficient is 0.684 , significance $(p)$ is 0.001 , which are within standard, and shows that when the insurance practitioners discovered that the insurance dynamic payment mechanism increases their living convenience, then their attitude towards use becomes more positive. Based on this, hypothesis $\mathrm{H}_{2}$ is established. Next, the insurance practitioners' attitude towards use of the insurance dynamic payment mechanism has a positive influence on the behavioral intention to use, and its standardized regression coefficient is 0.373 , significance $(p)$ is 0.019 , which are within standard; this shows that when the insurance practitioners' attitude towards the use of the insurance dynamic payment mechanism becomes better, their behavioral intention to use becomes concurrently stronger. Based on this, hypothesis $\mathrm{H}_{4}$ is established. It is clear that the insurance practitioners' perceived usefulness of the insurance dynamic payment mechanism has a positive influence on the behavioral intention to use. The standardized regression coefficient is 0.420 , significance $(p)$ is 0.01 , which are within standard, and shows that when the insurance practitioners recognized the convenience of using the insurance dynamic payment mechanism, then this would help increase the insurance practitioners' behavioral intention to use. Based on this, hypothesis $\mathrm{H}_{6}$ is established. Only $\mathrm{H}_{3}$ and $\mathrm{H}_{5}$ are not established in this study.

Table 3

Measurement Analysis Table of the Overall Theoretical Model

\begin{tabular}{|c|c|c|c|}
\hline \multicolumn{2}{|c|}{ Evaluation item } & Standard value & Research compatibility \\
\hline \multicolumn{4}{|c|}{ I. Basic Fit Standards } \\
\hline \multicolumn{2}{|c|}{ 1. Does not have negative error variance } & Yes & In line \\
\hline \multicolumn{2}{|c|}{ 2. Factor Load is between $0.5 \sim 0.95$} & Yes & In line \\
\hline \multicolumn{2}{|c|}{ 3. Does not have large standard errors } & Yes & In line \\
\hline \multicolumn{4}{|c|}{ II. Internal Fit } \\
\hline \multirow{2}{*}{\multicolumn{2}{|c|}{$\begin{array}{l}\text { 1. Composition Reliability CR (Measurement Model) of the Latent Variable } \\
\text { 2. Average Variability Extraction AVE (Measurement Model) of the Latent } \\
\text { Variable }\end{array}$}} & $>0.5$ & In line \\
\hline & & $>0.5$ & In line \\
\hline \multicolumn{2}{|c|}{ 3. Reliability of Each Item (Reliability Analysis) } & $>0.7$ & In line \\
\hline \multicolumn{4}{|c|}{ III. Overall Model Fit } \\
\hline \multirow{5}{*}{$\begin{array}{l}\text { Absolute Fit } \\
\text { Indices }\end{array}$} & 1. $\chi^{2} / d . f .($ Chi-square value/degree of freedom) & $1 \sim 5$ & 1.895; in line \\
\hline & 2. GFI (Goodness-of-Fit Index) & $>0.9$ or 0.8 & 0.891 ; in line \\
\hline & 3. $A G F I$ (Adjusted Goodness-of-Fit Index) & $>0.9$ or 0.8 & 0.846 ; in line \\
\hline & 4. $R M R$ (Root Mean Residual) & $<0.06$ & 0.037 ; in line \\
\hline & 5. RMSEA (Root Mean Square Error of Approximation) & $\begin{array}{l}<0.05 \\
(0.08 \text { Acceptable })\end{array}$ & 0.07 ; in line \\
\hline \multirow{2}{*}{$\begin{array}{l}\text { Incremental } \\
\text { Fit Indices }\end{array}$} & 6. NFI (Normed Fit Index) & $>0.9$ or 0.8 & 0.812 ; in line \\
\hline & 7. $C F I$ (Comparative Fit Index) & $>0.9$ or 0.8 & 0.899 ; in line \\
\hline \multirow{2}{*}{$\begin{array}{l}\text { Streamlined } \\
\text { Fit Indices }\end{array}$} & 8. $P N F I$ (Parsimony Normed Fit Index) & $>0.5$ & 0.65 ; in line \\
\hline & 9. PCFI (Parsimony Comparative Fit Index) & $>0.5$ & $0.719 ;$ in line \\
\hline
\end{tabular}




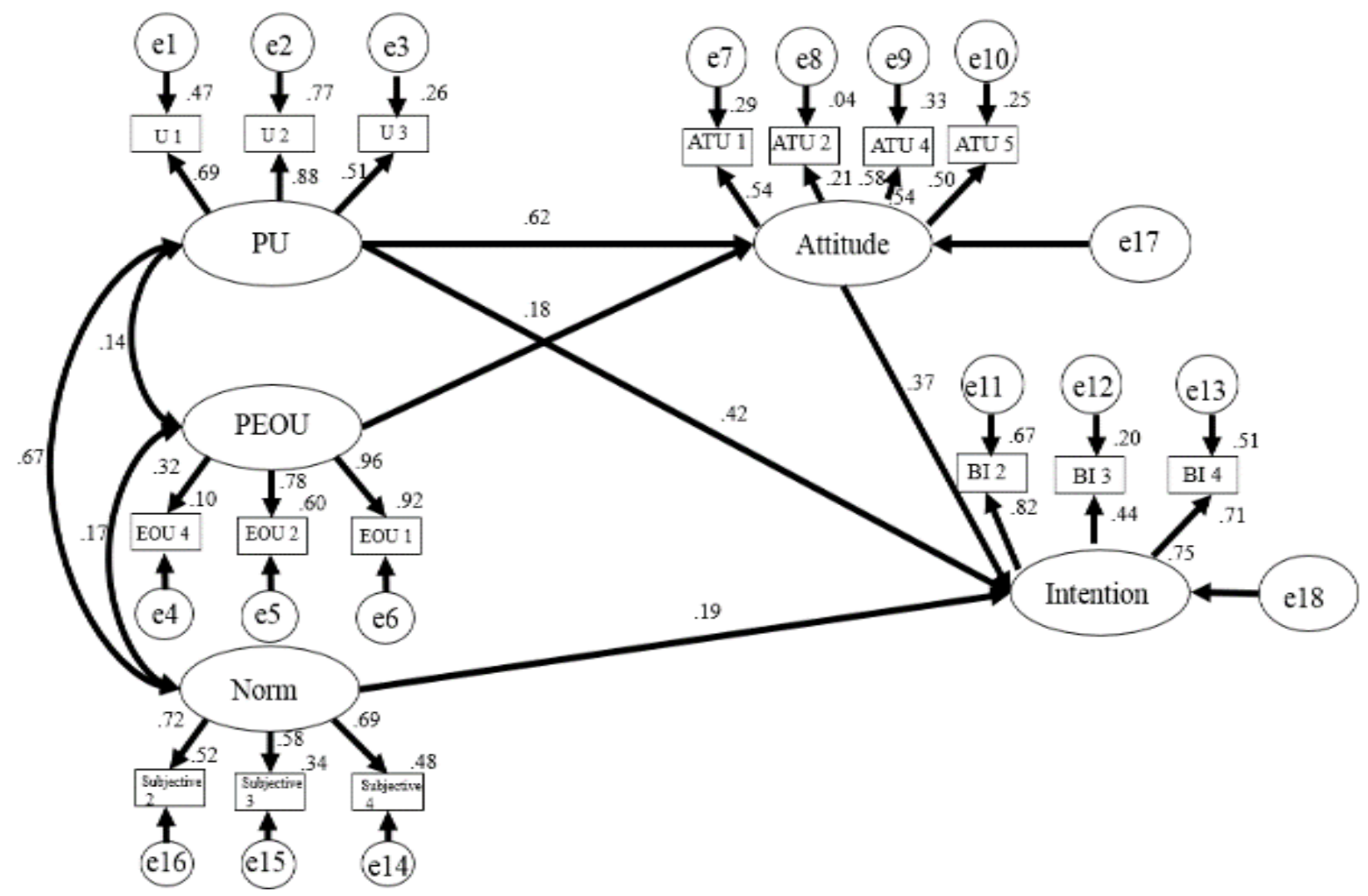

Figure 3. Overall path analysis of insurance practitioners.

e1: Usefulness 1

e2: Usefulness 2

e3: Usefulness 3

Perceived Usefulness points to Usefulness 1, Usefulness 2, and Usefulness 3

e4 points to Ease of Use 4

e5 points to Ease of Use 2

e6 points to Ease of Use 1

Perceived Ease of Use points to Ease of Use 4, Ease of Use

2, and Ease of Use 1

e16 points to Subjective 2

e15 points to Subjective 3

e14 points to Subjective 4
Norm points to Subjective 2, Subjective 3, and Subjective 4 e7 points to Attitude Towards Use 1 e8 points to Attitude Towards Use 2 e9 points to Attitude Towards Use 4 e10 points to Attitude Towards Use 5

Attitude points to Attitude Towards Use 1, Attitude Towards Use 2, Attitude Towards Use 4, Attitude Towards Use 5

e11 points to Behavioral Intention to Use 2

e12 points to Behavioral Intention to Use 3 e13 points to Behavioral Intention to Use 4 Intention to Use points to points to Behavioral Intention to Use 2, points to Behavioral Intention to Use 3, points to Behavioral Intention to Use 4

\section{Table 4}

Insurance Practitioners' Path Coefficient and Statistics

\begin{tabular}{|c|c|c|c|c|c|c|}
\hline Hypothesis & Internal variable & & External variable & $\begin{array}{l}\text { Regression } \\
\text { coefficient }\end{array}$ & $\begin{array}{l}\text { Standardized } \\
\text { regression } \\
\text { coefficient }\end{array}$ & Significance \\
\hline $\mathrm{H} 2$ & Attitude towards Use & $\leftarrow$ & Perceived Usefulness & 0.514 & 0.684 & $0.001 * * *$ \\
\hline H3 & Attitude towards Use & $\leftarrow$ & Perceived Ease of Use & 0.211 & 0.183 & 0.065 \\
\hline $\mathrm{H} 4$ & Behavioral Intention to Use & $\leftarrow$ & Attitude towards Use & 0.523 & 0.373 & $0.019 * *$ \\
\hline H5 & Behavioral Intention to Use & $\leftarrow$ & Subjective Norm & 0.163 & 0.191 & 0.097 \\
\hline H6 & Behavioral Intention to Use & $\leftarrow$ & Perceived Usefulness & 0.443 & 0.42 & $0.01 * *$ \\
\hline
\end{tabular}

${ }^{*} p<0.05$;** $p<0.01 ; * * * p<0.001$. 


\section{Conclusion and Proposal}

\section{Research Conclusion}

By analyzing the descriptive dimensions of the insurance practitioners in chapter four, on the subject of "I think we should use the measured health data from wearable devices, and use the constructed health assessment indicators to submit my health insurance premiums," the measured average number is 4.49 , which is lower than the general public's measured value. Therefore, we can conclude that on the research question of "Will using the wearable devices to construct the health assessment indicators be accepted or not", the insurance practitioners still prefer to construct the health assessment indicators on their own, and their acceptance of using wearable devices to perform the task is not high.

On the subject of "I think professionals using big data to process health assessment indicators and health insurance dynamic payment has value," the average number obtained is 5.15, which is the highest in the perceived usefulness dimension, and it can answer this study's question of "Will the creation of the health insurance dynamic payment mechanism through health management, insurance payment management and big data be accepted or not."

On the subject of "I will be willing to use the health assessment indicators and health insurance dynamic payment resulting from the big data processed by professionals," the average number is 4.64 , which is lower than general public's measured value. Therefore, on the question of "Will the use of big data to process health assessment indicator analysis and creation, and dynamically classify insurance payment be accepted," when it comes to using big data to process health assessment indicator analysis and creation, and dynamically classify insurance payment, the insurance practitioners are still conservative in the behavioral intention to use. Table 5 shows the results of this study's empirical analysis and data analysis.

Table 5

Results of This Study's Dimension Hypotheses (Insurance Practitioners)

\begin{tabular}{ll}
\hline Hypotheses of the study & Establish/Do not establish \\
\hline H1: The user's "perceived ease of use" of the health insurance dynamic payment & Establish \\
mechanism has a positive influence on the "perceived usefulness." & Establish \\
H2: The user's "perceived usefulness" of the health insurance dynamic payment & \\
mechanism has a positive influence on the "attitudes towards using." & H3: The user's "perceived ease of use" of the health insurance dynamic payment \\
mechanism has a positive influence on the "attitude towards using." & Do not establish \\
$\begin{array}{l}\text { H4: The user's "attitude towards using" the health insurance dynamic payment } \\
\text { mechanism has a positive influence on the "behavioral intention to use." }\end{array}$ & Establish \\
$\begin{array}{l}\text { H5: The user's "subjective norm" towards the health insurance dynamic payment } \\
\text { mechanism will positively influence the "behavioral intention to use." }\end{array}$ & Do not establish \\
$\begin{array}{l}\text { H6: The user's "perceived usefulness" towards the health insurance dynamic } \\
\text { payment mechanism has a positive influence on the "behavioral intention to use." }\end{array}$ & Establish \\
\hline
\end{tabular}

Explanation on the hypotheses that were not supported:

H3: The degrees of the insurance practitioners' perceptions of the health insurance dynamic payment mechanism are not easily implemented, thus, it is not considered a replacement of the unreasonable payment method of the national health insurance and commercial health insurance. Therefore, hypothesis three of this study is not supported.

H5: Insurance practitioners still place their own preference ahead when it comes to health insurance dynamic payment method, and will not be influenced by people who are important to them or influential to accept this mechanism. Therefore, hypothesis five of this study is not supported. 


\section{Research Contribution}

Currently, the premium payment amount remitted to the National Health Insurance is based on "monthly real wages," while the commercial health insurance uses consumer age as the basis for the premium amount charged. Since health wearable devices can obtain our health data in real time and in detail, the medical insurance costs that we need to pay everyday can be determined by the health assessment indicators determined by wearable devices on our bodies.

Angela McIntyre, Research Director at Gartner, said that fitness enthusiasts comprise the largest consumer group of wearable device products, she estimated that in 2016, the worldwide fitness and personal health wearable electronic products will grow up to five billion US dollars.

Currently, consumer electronics product manufacturers and sporting goods manufacturers who have added innovative wearable electronic devices into their product line are establishing a wearable electronic product ecosystem to strengthen their brands. Nike introduced Nike+Fuel Band in 2012. Their main target is to provide activity recording and analysis to users. Since this product is in line with Nike's sports products, therefore, its brand image and value are also reflected in the product, causing it to immediately become a popular product, and bringing about a boom in related sports products.

Aside from tracking our physical activities, in the future, we can use wearable devices to monitor our health status anytime and anywhere, through the measured health assessment indicators related to blood sugar, blood oxygen, and others. The measured data can also be used to assess whether we are healthy or not, and how much insurance premium we need to pay. Therefore, in order to preserve one's health and to avoid unnecessary insurance payments, the concept of using wearable devices and health assessment indicators to pay insurance premium is utilized. As a result, the general public will value their health even more. Of course, this will also promote the growth of the health food industry.

This study's innovative concepts not only serve as valuable references to succeeding research, but also contribute to actual practice. In the promising field of medical biotechnology, fitness, sports and health, and other related industries, all of them will benefit from this study's results.

\section{Research Proposal}

In the US, before wearable devices are launched in the market, they need to undergo FDA certification, but Taiwan does not yet have any similar legal or regulatory provisions. Aside from the key barriers in technology that need to be overcome, another problem faced by wearable medical devices is how to resolve its reliability issues. Currently, most of the companies that are in the business of health and medical wearable devices are technology companies, so the wearable devices are developed based on the engineers' thoughts and ideas, and there are no participating doctors or medical professionals in the development process. As a result, when the technological elements and concepts are being discussed, they fail to consider their reliability when used in the health and medical devices.

Therefore, we propose that wearable device businesses focus on the direction of long-term health monitoring, overcome battery life, medical accuracy and other such general concerns, and help the government in terms of regulatory provisions. All these will help Taiwan to greatly improve the medical model and insurance payment method.

When massive amount of health data encounter funding laws, data management and data leakage protection will also become important focus areas in health management. In the midst of all the big data, when 
problems arise, they are not necessarily caused by data volume, but may be instead due to the numerous platforms available, that contribute to the data leakage problem. Some of these include virtualization, cloud, and portable devices, and all these could greatly increase the risk of data leakage.

Therefore, when the health management centers collect the health data from the wearable devices, the areas that they need to consider are not limited to data creation, analysis and protection, but should also integrate different virtual and cloud platforms, so as to ensure data management security and prevent data leakage.

The health management requirements of clients today are continually growing, and they are focusing more on medical cost reimbursements, health needs protection, and other related problems. If there is insufficient health management centralized planning, then naturally, the general public will hesitate in realizing the necessity and urgency of enjoying the benefits of health management. Unavailable also will be improved mechanisms that would encourage consumers to pay attention to their daily health.

From the health management center's perspective, there is a need to establish the requirements before, during, and after the whole process of management and service, so as to be able to satisfy the general public's more pressing demand for health services. Through the health data collected from the wearable devices, they can provide professional health services, increase the effect of risk control and client satisfaction; on the other hand, they provide professional health treatment risk control, so that the services are more well-rounded, rational, and targeted, and provide a strong protection.

Insurance practitioners are still in the early phases of using big data, and they face many challenges such as weak underlying data, shortage of professionals, and a need to change the traditional business model. Therefore, insurance practitioners can utilize different algorithms to perform analysis on the health data collected from the wearable devices, use the analysis results to search for client groups that have latent insurance needs, and more accurately reflect the insured's specific insurance product needs and the preferred sales channel to be used. This will not only help the sales staff to become providers of insurance service that would increase the insured's experience, it can also be used as reference for designing new insurance products.

\section{References}

Ajzen, I. (1985). From intentions to actions: A theory of planned behavior. In J. Kuhl and J. Beckman (Eds.), Action control: From cognition to behavior. Heidelberg: Springer.

Bagozzi, R. P. (1981). Attitudes, intentions, and behavior: A test of some key hypotheses. Journal of Personality and Social Psychology, 41(4), 607-627.

Bagozzi, R. P., \& Yi, Y. (1988). On the evaluation of structural equation models, Academic of Marketing Science, 16(1), 76-94.

Bai, D. W., You, K. X., \& Chu, T. (2013). Google glass, smart watch will revolutionize smart wearable devices technology. TRI Topology Research Institute.

Buhulu. (2014). Apple and Duke University jointly developed healthKit. Retrieved from http://cner.com/por tal.php? $\bmod =$ view\&aid= 26735

Candice. (2014). Samsung announced during San Franciso general assembly that their wearable device simband is in progress. Retrieved from http://www.saydigi.com/2014/05/samsung-simband.html

Chen, K. (2012). Big data will soon drive the insurance industry's service outsourcing. Retrieved from http://chinasourcing.mofcom.gov.cn/c/2013-08-07/155107.shtml

Chen, K. H. (2000). A study on the factors that influence commercial insurance purchase. Feng Chia University Insurance Degree Master's Thesis.

Chen, S. Y., Lin, J. R., \& Tsai, Y. M. (2004). Making your first investment. The Complete Guide to Insurance and Finance, 17, 30-31. 
Chuang, P. N. (2014). A first in financial stocks! Cathay life insurance products merge with health management. China Times.

Davis, F. D. (1989). Perceived usefulness, perceived ease of use and user acceptance of information technologies. MIS Quarterly, 13(3), 319-340.

Gilbert. (2014). Google fit is android's answer to exercise and health tracking. Retrieved from http://www.engadget.com/ 2014/06/25/google-fit/

Hsieh, Z. M., Lin, Z. L., \& Yang, J. N. (2014). Key elements help wearable medical devices become the trend. New Electronics Technology Magazine, February, 28-42.

Hu, S. Z. (2013). Cloud era killer apps: Big data massive data analysis. World Magazine.

Huang, J. S., \& Chen, J. S. (2007). Health management theory and practice. Chinese Journal of Health Management, 1, 8-12.

Huang, W. Z. (2013). Analysis on the evolution and development strategy of wearable products. MIC Market Intelligence \& Consulting Institute.

Health Promotion Administration, Ministry of Health and Welfare. (2015). http://health99.hpa.gov.tw/OnlinkHealth/Onlink BMI.aspx

Kudo, K. (2010). Do your internal organs need to lose weight? Inconspicuous is more dangerous! Visceral fat accumulation is the invisible killer. Taipei: Faces Publishing Ltd.

Li, Y. F. (2011). Health insurance-The driving force of health management, Information China-e-Healthcare, 3, 36-37.

Li, S. L. (2014). Chang Gung University and compal enter into health care. China Times.

Li, H. Y. (2015). Understanding big data. Digital Age, 251, 62-67.

Li, S. W. (2008). Health insurance, buying right and inexpensive. Taipei, Cite Publishing Ltd.

Mathieson, K. (1991). Predicting user intentions: Comparing the technology acceptance model with the theory of planned behavior. Information Systems Research, 2(3), 173-191.

Moon, J. W., \& Kim, Y. G. (2001). Extending the TAM for a world-wide-web context. Information and Management, 38, 217-230.

Pan, X. (2014). Risk management research on commercial health insurance of China-From the perspectives of product and health management. School of International Trade and Economics, University of International Business and Economics Doctorate Thesis.

Roger, E. M. (1983). The diffusion of innovations (3rd ed.). New York: Free Press.

Shimp, T. A., \& Kavas, A. (1984). The theory of reasoned action applied to coupon usage. Journal of Consumer Research, 11(3), 795-809.

$\mathrm{Su}$, H. (2014). Health data services battle Google's release of Google fit. Epoch Times.

Taylor, S., \& Todd, P. A. (1995). Decomposition and cross effects in the theory of planned behavior: A study of consumer adoption intentions. International Journal of Research in Marketing, 12, 137-155.

Villasenor, J. F. (2014). Wearable device development can speed up the entry of health and fitness in the internet age. Retrieved from http://www.2cm.com.tw/technologyshow_content.asp?sn=1402260012

World Health Organization. (1946). WHO definition of health, preamble to the constitution of the World Health Organization. Adopted by the International Health Conference, New York, 19-22, June 1946; signed on 22, July 1946 by the representatives of 61 States (Official Records of the World Health Organization, No. 2, p. 100) and entered into force on 7 , April 1948.

Yang, R. L. (2004). A study on the strategic factors that influences consumers to buy insurance-Using Greater Taipei as example. Fu Jen Catholic University Applied Statistics Degree Master's Thesis.

Zhang, Z. C. (2014). Cathay life insurance and Taipei Medical University build remote health care service. China Times.

Zhan, E. T. (2008). A study on the relationship between different body types and health assessment indicators-Risk indicators of hidden obesity. Taipei Medical University School of Nutrition and Health Sciences Research Center Master's Thesis.

Zheng, X., Huang, Z. H., \& Zhang, Y. T. (2014). Data analysis helps insurance industry reach new revenue heights, Deloitte Monthly, September, 28-31. 